\title{
SPATIALLY PERIODIC STRUCTURES, UNDER FEMTOSECOND PULSED EXCITATION OF CRYSTALS
}

\author{
Evgueni F. Martynovitch \\ Irkutsk Filial of the Institute of Laser Physics, Siberian Branch of Russian Academy of \\ Sciences, Lermontov St. 130a, 664033, Irkutsk, Russia
}

Guillaume Petite

Laboratoire des Solides Irradiés, Commissariat à l'Energie Atomique/Direction des Sciences de la Matiére, Centre National de la Recherche Scientifique (UMR 7642) and Ecole Polytechnique, 91128, Palaiseau Cedex, France

Vladimir P. Dresvianski, Anton A. Starchenko Irkutsk State University, 20, Gagarin Blvd, Irkutsk, 664003, Russia

\begin{abstract}
Measuring the luminescence intensity of specially prepared irradiation defects induced in crystals, we observe that the longitudinal structure of quasi-interferences induced by two orthogonally polarized femtosecond pulses propagating together with different velocities is insensitive to the spatial broadening due to velocity dispersion in the crystals. On the contrary, it does depend on the pulse duration when it is changed by varying the spectral width of the radiation. It thus allows a direct measurement of the coherence time of such pulses. Stability of the axial selectivity is a good sign, taking away a number of serious limitations concerning possible applications.
\end{abstract}


In optics and quantum electronics, there is an increasing use of stationary as well as transient spatially periodic structures induced in optical crystals which can, for instance, diffract or back-reflect light and produce many other phenomena. Such structures can, of course, be produced through interferences, but an alternate method, exposed in [1,2], uses the superposition of two e.m. waves with orthogonal polarizations, providing a possibility of periodic modulations of light absorption by oriented quantum systems able to interact with both of the waves. Such a possibility of inducing localized periodic optical structures was demonstrated in cubic crystals in the case of counterpropagating femtosecond pulses [3].

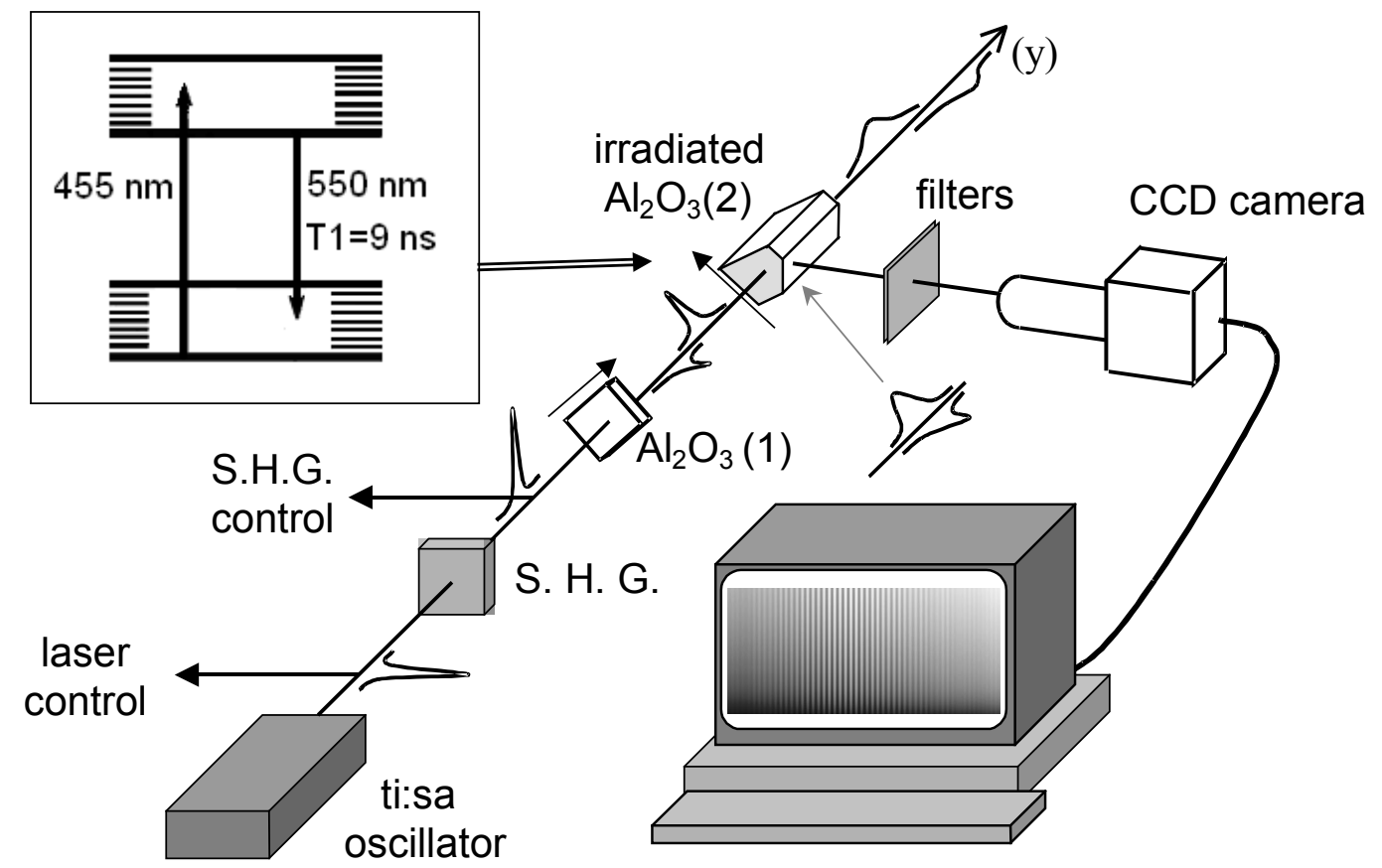

Figure 1 - Experimental set-up

In this paper, we study the possibility of inducing such polarization-induced periodic structures using copropagating ultrashort pulses. The experiment, depicted on figure 1, works as follows: the frequency doubled output of a femtosecond titanium-doped sapphire laser ( $400 \mathrm{~mW}$ average power, $65 \mathrm{MHz}$ repetition rate, $\approx 40$ fs pulses at $800 \mathrm{~nm} ; 18 \mathrm{~mW}$ averaged power at $400 \mathrm{~nm}$ ) is sent through a first sapphire crystal (crystal 1 on fig. 1). The frequency doubled beam being polarized at $45^{\circ}$ with respect to the optical axis of crystal 1 (indicated by the arrow on fig. 1), it produces at the crystal output two orthogonally polarized pulses, which are time delayed (by typically $125 \mathrm{fs}$ for a $5 \mathrm{~mm}$ crystal), due to the ordinary/extraordinary light velocity mismatch. These two pulses, mildly focused down to a diameter of $0.5 \mathrm{~mm}$, then enter a second sapphire crystal $\left(\mathrm{n}^{\circ} 2\right.$ on fig 1 ) whose optical axis is perpendicular to that of crystal 1 . In crystal $2(\approx 3 \mathrm{~cm}$ long), the two pulses thus exchange their velocities compared to crystal 1, catch up each other and fully overlap at a distance from the entrance face exactly equal to the thickness of crystal 1, and then separate from each other again.

Prior to the experiment, crystal 2 was irradiated in a fast-neutron reactor, thus producing luminescent centers with a broadband luminescence in the green-yellow region ( $550 \mathrm{~nm}, 9 \mathrm{~ns}$ ), together with an equally broad absorption band peaked at a wavelength of 455 $\mathrm{nm}$, with an absorption tail extending into the near-UV region. Moreover, one knows that their absorption is electric-dipolar, the responsible dipoles being oriented at approximately 40 degrees with respect to the crystal's optical axis [1].

Let us analyze the expected dependence of the luminescence signal as a function of the distance of observation to the entrance face. All processes are linear, and therefore the 
luminescence intensity is essentially determined by the absorbed intensity, i.e. is proportional to the exciting pulse intensity and to the square cosine of the angle between the pulse polarization and the absorbing dipole orientation. In the region where the pulses do not overlap, there is no spatial structure of the luminescence intensity other than a slow exponential decrease due to the pulses' intensity attenuation as they propagate into the crystal. Both pulses contribute practically equally since they are both polarized at nearly $45^{\circ}$ with respect to the absorbing centers. The situation becomes more intricate in the region where the pulses overlap since the resulting light polarization will progressively rotate from one pulse's polarization to the other's, thus inducing a time modulation of the excitation probability (it is the time integral of this probability that matters at a given point in space). This situation was theoretically analyzed in [4], yielding the following result: one should observe a spatially periodic modulation of the luminescence intensity, localized in the region where the two pulses overlap. The contrast of such oscillations is maximum when the pulses are polarized at $45^{\circ}$ with respect to the absorbing defect dipole (quite close to our experimental situation). The spatial period of the oscillations is equal to $\lambda / \Delta n$ ( $\lambda$ : vacuum wavelength, $\Delta n$ : ordinary/extraordinary index difference, equal to 0.008 for sapphire). In the case of an incoherent excitation (i.e. when the pulse duration $\tau_{\mathrm{p}}$ is larger than the phase relaxation time of the material), the width $\mathrm{Y}$ of the envelope of the modulations is uniquely determined by the duration of the exciting pulse (let us note here that the longitudinal relaxation time - 9ns - is much longer than the duration of the pulses we use).

However, such calculations assumed Fourier limited pulses. Meanwhile, in our experiment (like in any experiment involving propagation of ultrashort pulses in dispersive media) one has to consider the pulse lengthening due to Group Velocity Dispersion (GVD) during propagation through crystals 1 and 2, with the consequence that the pulses that overlap in crystal 2 are no longer Fourier limited. One would expect that the width $\mathrm{Y}$ is increased by an amount corresponding to the pulse duration increase by GVD (the "chirp") due to propagation through twice the thickness of crystal 1, but no real theoretical evaluation of this increase of $Y$ for non-Fourier limited pulses is presently available.

Other important experimental details are the following: the spectral coherence of the fundamental laser pulse is controlled with a combined measurement of the pulse duration (using an autocorrelator) and of the frequency spectrum. Frequency doubling is achieved in a Type I, $100 \mu \mathrm{m}$ thick BBO crystal. The spectrum of the frequency doubled pulse is checked using a TRIAX 180 spectrometer, but we do not have at our disposal means to measure directly the duration of the second harmonic pulse, which we assume to be in the ratio $\sqrt{2}$ with the fundamental pulse duration (which amounts to assuming a gaussian line shape and neglecting the doubling crystal GVD at the second harmonic wavelength). The fundamental spectrum can be controlled by adjusting the prisms composing the intra-cavity GVD compensation line.

Crystal 2 is cut according to a special shape (see fig. 1) allowing imaging of the luminescent zone excited along the propagation channel perpendicularly to the laser propagation direction. Scattered second harmonic light is filtered out using a low-pass colored glass filter, and imaged using a microscope objective on a 1024x256 pixel $^{2}, 26 \mu \mathrm{m}$ pixel size, $\mathrm{CCD}$ array cooled down to $-70^{\circ} \mathrm{C}$, for noise reduction. The integration time is $32 \mu \mathrm{s}$. The images of the luminescence distribution are then treated numerically to extract relevant quantities about this distribution, as shown on figure 2 .

Curve (a) shows the result of a summation of the pixel contents over pixel columns, which reflects the luminescence intensity dependence along the laser propagation direction. One clearly observes the two expected features: a slow exponential decrease of the luminescence intensity due to crystal absorption, and superposed "high frequency" oscillations in the region where the pulses overlap. This high frequency component is extracted using a numerical high-pass filtering technique, and shown on curve (b). The 
resulting distribution is then fit to a sinusoidally modulated gaussian distribution, and the FWHM of its envelope is taken as the width Y of the periodic structure. One spatial period covers 13 pixel columns, which translates in the object plane into $\approx 50 \mu \mathrm{m}$, in quite good agreement with the expected value at a $400 \mathrm{~nm}$ wavelength.

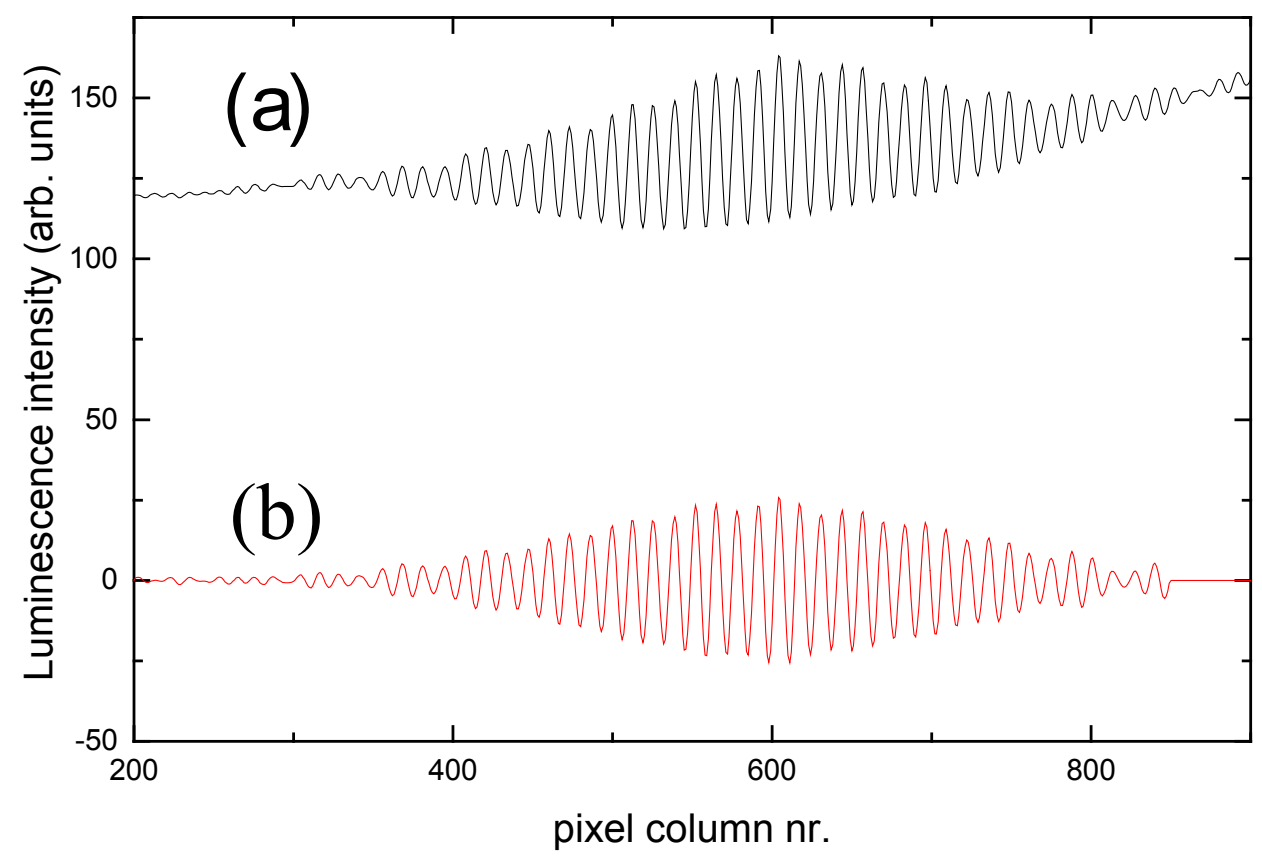

Figure 2 - Spatial dependence of the luminescence intensity. (a) raw data (summed over pixel columns) (b) after numerical extraction - by high pass filtering - of the high frequency part.

To investigate the effect of GVD on the luminescence intensity distribution, and more exactly on the width of the spatially periodic structure, such an experiment was reproduced using two different thickness of crystal $1(5 \mathrm{~mm}$ and $16 \mathrm{~mm}$, the total GVD induced in the overlap region corresponding to twice the thickness of crystal 1).

Table I: Time and spectral characteristics of the pulses used in the experiment. $\tau_{L}$ : initial pulse duration, $\Delta \lambda$ :measured spectral width at $400 \mathrm{~nm}, \tau_{p}$ : estimated chirped pulse duration. The spectral widths are corrected from the spectrometer limited resolution $(\approx 1 \mathrm{~nm})$. All values quoted are FWHM of intensity.

Configuration

$\tau_{\mathrm{L}}(800 \mathrm{~nm})$

$\tau_{\mathrm{L}}(400 \mathrm{~nm})$

$\Delta \lambda(400 \mathrm{~nm})$

$\tau_{\mathrm{p}}(400 \mathrm{~nm}$, chirped $)$

$\tau_{\mathrm{p} .} \Delta \mathrm{v}(400 \mathrm{~nm}$, chirped $)$
(1)

$26.5 \mathrm{fs}$

$19 \mathrm{fs}$

$12.5 \mathrm{~nm}$

$88 \mathrm{fs}$

$\approx 2 \approx 5.5$

(2)

$26.5 \mathrm{fs}$

$19 \mathrm{fs}$

$12.5 \mathrm{~nm}$

$240 \mathrm{fs}$

Table I summarizes the experimental conditions for the two configurations. The figures quoted for the chirped pulse durations are calculated using the spectral width and the dispersion characteristics of sapphire. The consistency of such estimations was checked by 
simultaneous measurements of the pulse duration (in the IR) and the spectral width. Concerning the initial IR pulse used in the luminescence measurements, we measure a duration of $43 \mathrm{fs}$ and a spectral width of $35 \mathrm{~nm}$ (all values FWHM of intensity), which is close to twice the Fourier limit. This can be explained by the fact that our autocorrelator is not equipped with a compensating plate, which limits the minimum measurable duration to about 30 fs. Assuming a gaussian Fourier limited pulse results in a $26.5 \mathrm{fs}$ pulse, which seems quite reasonable and yields a pulse duration at the second harmonic wavelength of $19 \mathrm{fs}$, in fair agreement with the measured $13.5 \mathrm{~nm}$ spectral width (corresponding Fourier limit: $17.5 \mathrm{fs}$, before correction from the spectrometer resolution $: \approx 1 \mathrm{~nm}$ ). Then, using the maximum bandwidth available in the IR $(70 \mathrm{~nm}$, which we did not use in the luminescence experiment since the acceptance of the BBO crystal does not allow to fully frequency double it) we measured the chirped pulse duration to be $73 \mathrm{fs}$ and $222 \mathrm{fs}$ respectively for configurations 1 and 2, to compare with the estimated chirps of respectively 50 and $215 \mathrm{fs}$.

The conclusion of such measurements is that, despite a considerable lengthening of the pulse for configuration 2 compared to 1 , we do not observe any dependence of the width of the envelope of the oscillating longitudinal structure upon the chirped pulse duration.

Table II : Time and spectral characteristics of the pulses used in the experiment with a variable spectral width, and comparison of the measured spatial profile width (Y) with the calculated values. The spectral widths are corrected from the spectrometer's limited resolution $(\approx 1 \mathrm{~nm})$. All values quoted are FWHM of intensity.

\begin{tabular}{llll} 
Configuration & $(\mathrm{a})$ & $(\mathrm{b})$ & (c) \\
$\tau_{\mathrm{L}}(800 \mathrm{~nm})$ & $26.5 \mathrm{fs}$ & $50 \mathrm{fs}$ & $26.5 \mathrm{fs}$ \\
$\tau_{\mathrm{L}}(400 \mathrm{~nm})$ & $19 \mathrm{fs}$ & $35 \mathrm{fs}$ & $19 \mathrm{fs}$ \\
$\Delta \lambda(400 \mathrm{~nm})$ & $12.5 \mathrm{~nm}$ & $6.8 \mathrm{~nm}$ & $12.5 \mathrm{~nm}$ \\
$\tau_{\mathrm{p}}(400 \mathrm{~nm})$ & $88 \mathrm{fs}$ & $99 \mathrm{fs}$ & $240 \mathrm{fs}$ \\
$Y(\mathrm{~mm})$ & $1 \pm 0.1$ & $2 \pm 0.1$ & $1 \pm 0.1$ \\
$c \tau_{L} / \Delta n(\mathrm{~mm})$ & 0.70 & 1.3 & 0.70 \\
$c \tau_{p} / \Delta n(\mathrm{~mm})$ & 3.4 & 3.9 & 9.3 \\
\hline
\end{tabular}

It is also possible to change the pulse duration, staying Fourier limited, by varying the spectral width of the exciting pulse. The result and experimental conditions of such an experiment are summarized in Table II. Three configurations were compared: (a) identical to configuration 1 above, (b) as (a), but using a "narrow-band" spectrum $(\Delta \lambda=7.8 \mathrm{~nm}$ instead of 13.5) and (c) as (a), but inserting a $4.5 \mathrm{~cm}$ silica rod between crystals 1 and 2, which produces a chirp of 225 fs (adding to the $50 \mathrm{fs}$ chirp induced by $1 \mathrm{~cm}$ of sapphire). In this case, the expected corresponding variation of the spatial width of the profile between configurations (a) and (b) was observed: the relation $\mathrm{Y}_{\mathrm{a}} / \mathrm{Y}_{\mathrm{b}}=\Delta \lambda_{\mathrm{a}} / \Delta \lambda_{\mathrm{b}}$ is rather accurately checked $\left(\mathrm{Y}_{\mathrm{a}} / \mathrm{Y}_{\mathrm{b}}=\right.$ $2 \pm 0.2$, while $\left.\Delta \lambda_{\mathrm{a}} / \Delta \lambda_{\mathrm{b}}=1.85 \pm 0.2\right)$. Meanwhile, In case (c), despite a considerable increase of the chirped pulse duration, the spatial width of the structure is identical to that of case (a), which is consistent with the result of the first experiment (no effect of the GVD on Y). The data obtained is also in reasonable agreement with the calculated width of the profile $\mathrm{Y}=\mathrm{c} \tau_{\mathrm{c}} / \Delta \mathrm{n}$, where $\tau_{\mathrm{c}}$ is the longitudinal coherence time (assumed equal to $\tau_{\mathrm{L}}$ ). The calculated widths are somewhat smaller, probably a sign that the gaussian pulse assumption used for the pulse duration estimates is not excellent. If one uses $\tau_{\mathrm{p}}$ instead of $\tau_{\mathrm{L}}$, the values are on the contrary very far from the measured ones, and do not even behave qualitatively the same. Contrary to the chirped pulse duration, the longitudinal coherence time is not affected by the spatial dispersion and broadening of the femtosecond pulses propagating through the crystals. 
That is why the periodic structures induced for a given spectral width are all of the same width. Our conclusion is thus that the spatial width of the periodic structures generated by our "polarization rotation" mechanism is not affected by the broadening of the femtosecond pulses due to GVD, but is dependent on their longitudinal coherence time only. This stability and independence of GVD can be considered as a good sign, taking away a number of serious limitations concerning possible applications.

The authors are grateful to academician S.N.Bagaev for his interest in this work and enlightening discussions of its results. The present work has been partially supported by the Russian Fund for Basic Research (grant RFBR n 01-02-690a), by the University of Russia Program (grant UR.01.01.009) and by the French MAE/DRI of CEA.

\section{References}

1. Martynovitch E.F., JETP Letters 49, 655-658 (1989)

2. Martynovitch E.F., Letters of J. Tech. Phys., 15, 60-64 (1989)

3. Kozlov D.A. and Martynovitch E.F., « Excitation of photoluminescent crystals of high and low symmetry by encountering femtosecond pulses ». Proceedings of the 4rth AllRussian school-seminar «Luminescence and related phenomena ». Irkutsk University. 141149 (2001).

4. Martynovitch E.E., Martynovitch E.F. and Polityko S.I.; Optics and Quantum Electronics, 27, 725-734 (1995) 